\title{
BMJ Open Cross-sectional survey for assessing cancer care providers' characteristics and attitudes on smoking cessation in Colombia and Mexico
}

\author{
Irene Tami-Maury (D , ${ }^{1}$ Laura Suchil, ${ }^{2}$ Luz Myriam Reynales-Shigematsu, ${ }^{3}$ \\ Leonor Garcia-Gomez, ${ }^{4}$ Mixing Chen, ${ }^{5}$ Sanjay Shete, ${ }^{5}$ Alejandro Betancur, ${ }^{6}$ \\ Paul M Cinciripini, ${ }^{5}$ Ernest Hawk, ${ }^{5}$ Hector Garcia ${ }^{6}$
}

To cite: Tami-Maury I, Suchil L, Reynales-Shigematsu LM, et al. Cross-sectional survey for assessing cancer care providers characteristics and attitudes on smoking cessation in Colombia and Mexico. BMJ Open 2021;11:e041447. doi:10.1136/ bmjopen-2020-041447

- Prepublication history for this paper is available online. To view these files, please visit the journal online (http://dx.doi org/10.1136/bmjopen-2020041447).

Received 09 June 2020 Revised 29 September 2020 Accepted 05 November 2020

Check for updates

(c) Author(s) (or their employer(s)) 2021. Re-use permitted under CC BY-NC. No commercial re-use. See rights and permissions. Published by BMJ.

For numbered affiliations see end of article.

Correspondence to

Dr Irene Tami-Maury;

Irene.Tami@uth.tmc.edu

\section{ABSTRACT}

Objective Cancer care providers' (CCPs) attitudes towards smoking cessation are influenced by many factors, including their smoking status and knowledge. Our objective was to assess CCPs' characteristics, tobacco use and smoking cessation practices in two Latin American cancer centres.

Design Cross-sectional survey.

Settings Two urban cancer centres located in Colombia and Mexico.

Participants A total of 238 CCPs.

Measures Online survey consisted of 28 close-ended questions adapted from the 2012 International Association for the Study of Lung Cancer survey and the Global Adult Tobacco Survey developed by the WHO. Means, frequencies and proportions were reported for each country. Factors associated to providing of smoking cessation treatment or referral at initial visit were evaluated using logistic regression.

Results Current smoking prevalence was $10.5 \%$ and $12.3 \%$ among Colombian and Mexican CCPs, respectively. Around three quarters of the Colombian (86.4\%) and Mexican CCPs $(66.1 \%)$ considered to have inadequate training in smoking cessation. Approximately two-thirds of Colombian (67.5\%) and Mexican CCPs (63.9\%) reported always or most of the time asking patients about tobacco use during the initial visit. In Colombia and Mexico, the most relevant barriers for providing cessation services were (1) difficulties for motivating patients with cancer, (2) patient resistance in quitting smoking, (3) lack of local resources or referral centres for smoking cessation and (4) lack of training in smoking cessation. CCPs appointed at Instituto Nacional de Cancerología were less likely to provide cessation treatment or referral to their patients if they had less than $50 \%$ of their time devoted to patient care and were former or current smokers. The regression model for Instituto de Cancerología did not retain statistically significant variables.

Conclusion Our findings highlight an urgent need for assisting Latin American CCPs in their quitting efforts as well as expanding formal smoking cessation training specifically tailored to these professionals for improving patients' cancer prognosis and quality of life.
Strengths and limitations of this study

- This study covered two renowned cancer centres in Latin America.

- Among the first reports in the region assessing characteristics, tobacco use and smoking cessation practices among cancer care providers.

- Participating institutions are not comparable as the Colombian cancer centre is a private institution, and the Mexican cancer centre is a public entity.

- Results are mostly limited to the cross-sectional design of the study and self-reported data, which are subjected to bias.

- Study findings may encourage future tobacco treatment training opportunities tailored to cancer care providers in Latin America.

\section{INTRODUCTION}

Cancer is now one of the most important causes of illness, disability and death among adults in Latin America, ${ }^{1}$ particularly those forms of cancer attributable to smoking. Smoking-related deaths in the region are closely associated with previous and current patterns of smoking prevalence ${ }^{2}$ with stages of the smoking epidemic varying from one country to another (eg, stage 1: $<20 \%$ smoking prevalence, mainly among men; stage 2: rapid increase in male smoking prevalence towards a peak of $40 \%$ to $80 \%$; stage 3: flattening of the smoking curve and some convergence of male and female smoking prevalence and stage 4: declined of smoking prevalence in both genders with the continued increase of deaths for a time, particularly among men). However, the anticipated mortality decline due to chronic conditions closely connected with smoking will slow down considerably if prevention and control strategies are not implemented and sustained over time, particularly among 
vulnerable groups. ${ }^{1}$ According to the 2019 WHO Report on the Global Tobacco Epidemic, ${ }^{3}$ the estimated prevalence of current cigarette smoking among those aged 15 years and older in Colombia is $13.0 \%$, with a higher prevalence among men (18.8\%). For Mexico, the prevalence is $14.3 \%$ for women and $21.9 \%$ for men.

Studies conducted in North America have shown that even after a cancer diagnosis, a significant number of patients continue to smoke ${ }^{45}$ despite the direct relationship between cigarette smoking and numerous adverse cancer treatment outcomes that negatively impact the overall survival rate. ${ }^{6}$ Along the same line, smoking behaviours of healthcare workers have been the topic of several studies because of the documented effectiveness of health professionals in precipitating patient smoking cessation and their ineffectiveness when the health professionals are smokers. ${ }^{7}$ In a systematic review of 229 studies representing 63 countries, the overall pooled prevalence of tobacco use among 457415 healthcare workers was $21 \% .{ }^{8}$ Findings from an online survey conducted in 2012 among 1507 members of the International Association for the Study of Lung Cancer (IASLC) revealed that $5 \%$ and $24 \%$ of the respondents were current and former smokers, respectively. ${ }^{9}$ Despite that a review of the trials reported by the Cochrane Tobacco Addiction Group and six Latin American databases suggest that systematic identification of patients' smoking status and a brief simple advice routinely offered by their physicians will benefit motivated smokers ${ }^{10}$ the credibility of such professionals is undermined by their smoking behaviour. ${ }^{11} \mathrm{~A}$ metaanalysis conducted from 1987 to 2010 found Mexico to have a high physician smoking prevalence rate (current smoking), ranging from $21 \%$ to $27 \% .{ }^{12}$ A study conducted in 1997 assessing the smoking prevalence among healthcare professionals appointed at the National Health Institutes in Mexico reported $42.5 \%$ of smoking prevalence specifically among cancer care providers (CCPs) ${ }^{13}$ These findings, however, should be interpreted with caution, as the survey response rate among this group of health providers was only $8 \%$. In Colombia, data linked to the prevalence of tobacco consumption among healthcare professionals are few and frequently controversial. A study conducted in 2013 among healthcare workers at a Colombian university revealed a smoking prevalence of nearly $12 \% .^{14}$

Continued tobacco use after cancer diagnosis often represents a combination of failure in raising awareness among patients about the benefits of quitting smoking and limited capacity among healthcare professionals to implement evidence-based smoking cessation interventions in cancer centres. Gaps in knowledge, attitudes and perceived self-efficacy (ie, ability of healthcare providers to counsel patients about smoking cessation) cannot be overlooked when considering further integration of cessation interventions into clinical practice. ${ }^{15}$ As substantial data have demonstrated in North America, ${ }^{6}$ a cancer diagnosis can be used as a window of opportunity to assist patients with cancer in their quitting process.
Unfortunately, integrating evidence-based smoking cessation interventions as a standard component of cancer care continues to be challenging for many cancer centres in Latin America, as barriers and facilitating factors for implementing such programmes have not yet been identified. Thus, our study aimed to assess CCPs' characteristics, self-reported tobacco use, knowledge and attitudes towards smoking cessation and the associated challenges for implementing such services in the Instituto de Cancerología (IDC) Las Americas Auna, Colombia; and in the Instituto Nacional de Cancerología (INCan), Mexico.

\section{METHODS \\ Study design and participants}

This cross-sectional study was conducted among 238 CCPs providing care at IDC and INCan. These Latin American institutions have large catchment areas with the potential to influence the implementation of evidence-based tobacco cessation programmes in the region because of their reputations. IDC is a private centre (physicianowned) in Medellin, Colombia, providing integral service in cancer education, prevention, diagnosis and treatment. Today, IDC is among the most influential cancer centres in Colombia, treating people from all over the country as well as from South America, Central America and the Caribbean. In 2015 (when this study was conducted), IDC had a total of 76536 physician consultations and 1022 hospitalisations.

On the other hand, INCan is a public health institution dedicated to the prevention, early detection, diagnosis and treatment of cancer in Mexico. INCan is the largest cancer treatment and research hospital in Mexico, serving 211235 patients through consultations and 7208 through hospitalisation in 2016. At present, INCan is the best equipped oncologic centre in Latin America.

\section{Procedures}

Psychosocial, behavioural and demographic information, as well as tobacco-related data, were collected through an anonymous online survey using the Qualtrics platform targeting a convenient sample of CCPs. Qualtrics is an information security tool chosen by the research team for its advanced design capabilities and its Health Insurance Portability and Accountability Act compliance. The target population (18 years of age or older) was physicians, nurses and other healthcare professionals providing direct care to patients with cancer at IDC or INCan. An invitational email with the embedded link to the online survey was sent to potential study participants. The survey remained open for 4 consecutive months (Colombia: September-December 2015; Mexico: April-July 2016), during which monthly reminder emails were sent out to increase the response rate.

The data collection instrument (online survey) consisted in 28 close-ended questions adapted from the 2012 IASLC $^{9}$ survey and the Global Adult Tobacco Survey $^{16}$ developed by the WHO for systematically 
monitoring adult tobacco use and tracking key tobacco control indicators across countries. The online survey included sociodemographic information such as age, gender, educational level, academic degree, years since graduation as well as CCPs' percentage of time exclusively devoted to patient care. All these characteristics were examined as categorical variables with the exception of age, which was treated as a continuous variable.

The online survey also included questions about cigarette smoking and the use of other tobacco products, including e-cigarettes. Prevalence of current cigarette smoking among respondents was determined by those CCPs who reported smoking 100 cigarettes in their lifetime and who were smoking cigarettes either everyday or some days at the time of completing the survey. Former smokers were those respondents who reported smoking at least 100 cigarettes in their lifetime, but who had quit smoking at the time of completing the survey. Never smokers were those study participants who never smoked or reported smoking less than 100 cigarettes in their lifetime. Current, former and never use of tobacco products other than cigarettes (eg, cigars, pipes, water pipes and/ or chewing tobacco) were also calculated. The status of e-cigarettes consumption among CCPs was assessed separately.

Six questions evaluated CCPs' knowledge and perceptions about tobacco use among their patients: (1) smoking is the main risk factor for lung cancer, (2) smoking negatively impacts cancer treatment and survivorship, (3) smoking cessation should be part of cancer treatment, (4) I have adequate smoking cessation training, (5) healthcare providers should be aware of new and emerging tobacco products and (6) healthcare providers should not smoke. Responses were provided on a 5-point Likert scale ranging from 'strongly disagree' to 'strongly agree', which also included a neutral 'no opinion' option.

Three questions evaluated CCPs' smoking cessation practices at initial visits: (1) I ask patients with cancer about tobacco use, (2) I counsel patients with cancer to quit smoking and (3) I provide treatment or refer patients with cancer to quit. Three questions evaluated CCPs' smoking cessation practices during follow-up appointments: (1) I ask patients with cancer about their tobacco use, (2) I ask whether patients with cancer had quit smoking and (3) I emphasise to patients the importance of quitting smoking. Response options ranged from 'never', 'rarely', 'sometimes', 'most times' and 'always' for both sets of questions.

A list of 11 potential barriers for providing smoking cessation services to their patients with cancer and survivors was provided to the study participants in the online survey: (1) difficulty for motivating patients with cancer, (2) smoking cessation among patients with cancer is a waste of time, (3) lack of time for smoking cessation counselling, (4) lack of reimbursement for smoking cessation services, (5) patients' resistance in quitting, (6) lack of training, (7) lack of local resources or referral centres for smoking cessation, (8) cost of cessation treatment, (9) unavailable smoking cessation medication, (10) tobacco use among healthcare providers and (11) marketing of new and emerging tobacco products as an alternative for smoking cessation. Responses were provided on a 5-point Likert scale ranging from 'completely disagree' to 'strongly agree' in addition to a 'no opinion' option.

\section{Data analysis}

Responses were collected anonymously, and once the data collection process was completed, data were imported from Qualtrics into Stata (V.15.0, College Station, Texas, USA: StataCorp LLC) for analysis purposes. CCPs not reporting dedication to patient care $(n=17)$ were excluded for analytic purposes. Study variables were summarised, in aggregate, using standard descriptive statistics such as mean, SD, frequency and proportion. Relevant variables were compared between cancer centres using $\chi^{2}$, Fisher's exact and two-sample t-tests, when appropriate. In an exploratory analysis, CCPs' characteristics and reported barriers were used to predict the odds of providing cessation treatment or referral to patients with cancer at the initial visit using logistic regression for each study site. We did not conduct any variable selection, such as stepwise selection, because such approaches are known to lead to biased coefficient estimation. ${ }^{17}$ The significant threshold was set at 0.05. All analyses were conducted using Stata V.15.1 (StataCorp, College Station, Texas, USA).

\section{Patient and public involvement}

Patients were not involved in the study design. Only cancer care providers participated in our research study.

\section{RESULTS}

\section{Response rates}

All the 112 CCPs at IDC eligible to participate in the study submitted a survey response. Ninety-four out of those 112 CCPs agreed to participate in the study, yielding a response rate of $83.9 \%$. At INCan, the survey was sent to 824 eligible CCPs. Out of the 193 CCPs who responded to the survey, 144 agreed to participate in the study $(17.5 \%$ response rate).

\section{Characteristics of the study sample and self-reported smoking status}

Table 1 summarises the main demographics and selfreported tobacco use prevalence among respondents. Among the 94 IDC CCPs (Colombia), the largest proportion of responses $(58.5 \%)$ was from women (mean age 41 years old), with those having a medical degree representing the primary respondent category $(46.8 \%)$. More than half of the sample (55.9\%) had more than 10 years since graduation with the vast majority $(80.9 \%)$ reporting to dedicate $50 \%$ or more of their time to patient care. Among those CCPs self-reporting smoking status, $10.5 \%$ and $22.4 \%$ were either current or former smokers, respectively. 
Table 1 Characteristics of the surveyed cancer care providers $(n=238)^{a}$

\begin{tabular}{llcc}
\hline Characteristic & IDC $\mathbf{N}=\mathbf{9 4} \mathbf{n}(\%)$ & INCan $\mathbf{N}=\mathbf{1 4 4} \mathbf{n}(\%)$ & P value \\
Mean age, years $( \pm \mathbf{S D})$ & $40.5( \pm 10.9)$ & $36.9( \pm 9.8)$ & 0.009 \\
Gender & & & \\
Female & $55(58.5)$ & $96(66.7)$ & 0.202 \\
\hline Male & $39(41.5)$ & $48(33.3)$ &
\end{tabular}

\section{Education level}

\begin{tabular}{|c|c|c|c|}
\hline High school/tech career & $23(24.5)$ & $14(9.8)$ & 0.001 \\
\hline Bachelors & $10(10.6)$ & $40(28.0)$ & \\
\hline Specialty & $1(1.1)$ & $50(35.0)$ & \\
\hline Masters & $3(3.2)$ & $23(16.1)$ & \\
\hline Doctorate & $16(17.0)$ & $13(9.1)$ & \\
\hline Other† & $41(43.6)$ & $3(2.1)$ & \\
\hline \multicolumn{4}{|l|}{ Professional degree } \\
\hline Medicine & $44(46.8)$ & $81(56.3)$ & 0.324 \\
\hline Nursing & $24(25.5)$ & $33(22.9)$ & \\
\hline Other $¥$ & $26(27.7)$ & $30(20.8)$ & \\
\hline \multicolumn{4}{|l|}{ Years since graduation } \\
\hline$\leq 10$ years & $41(44.1)$ & $34(23.6)$ & 0.001 \\
\hline$>10$ years & $52(55.9)$ & $110(76.4)$ & \\
\hline \multicolumn{4}{|c|}{ Time percentage devoted to patient care } \\
\hline$<50 \%$ & $18(19.1)$ & $52(36.1)$ & 0.005 \\
\hline$\geq 50 \%$ & $76(80.9)$ & $92(63.9)$ & \\
\hline \multicolumn{4}{|l|}{ Cigarette smoking status } \\
\hline Current smoker & $7(10.5)$ & $15(12.3)$ & 0.162 \\
\hline Former smoker & $15(22.4)$ & $42(34.4)$ & \\
\hline Never smoker & $45(67.2)$ & $65(53.3)$ & \\
\hline \multicolumn{4}{|c|}{ Other tobacco product use status } \\
\hline Current user & $1(8.3)$ & $3(9.7)$ & 0.769 \\
\hline Former user & $4(33.3)$ & $7(22.6)$ & \\
\hline Never user & $7(58.3)$ & $21(67.7)$ & \\
\hline \multicolumn{4}{|l|}{ e-cigarette use status } \\
\hline Current user & $2(3.5)$ & $1(1.1)$ & 0.573 \\
\hline Former user & $3(5.2)$ & $6(6.5)$ & \\
\hline Never user & $53(91.4)$ & $86(92.5)$ & \\
\hline \multicolumn{4}{|c|}{$\begin{array}{l}\text { Aware of local resources or services that } \\
\text { assist patients with quitting smoking }\end{array}$} \\
\hline No & $58(86.6)$ & 47 (37.3) & 0.001 \\
\hline Yes & $9(13.4)$ & $79(62.7)$ & \\
\hline
\end{tabular}

${ }^{*}$ Due to missing data, not all the variables add up to the total sample.

†Includes subspecialty, certificate.

łncludes cytotechnology, psychology, physiotherapy, nurse technician, auxiliary nurse, radiotherapy technologist.

IDC, Instituto de Cancerología; INCan, Instituto Nacional de Cancerología.

Among the 144 INCan cancer care providers (Mexico) who agreed to participate in the study, $66.7 \%$ were women (mean age 37 years old) and $56.3 \%$ had a medical degree. More than three quarters $(76.4 \%)$ of the study sample had more than 10 years since graduating from their terminal degree with $63.9 \%$ reporting to dedicate $50 \%$ or more of their time to patient care. Among INCan respondents, $12.3 \%$ and $34.4 \%$ reported being either current or former smokers, respectively. 
Table 2 Respondents' knowledge of tobacco use and tobacco cessation $(n=238)^{\star}$

\begin{tabular}{|c|c|c|c|}
\hline Knowledge/perceptions & IDC n (\%) & INCan n (\%) & $\mathbf{P}$ value \\
\hline \multicolumn{4}{|c|}{ Smoking is the main risk factor for lung cancer } \\
\hline Completely disagree/disagree/no opinion & $9(13.6)$ & $11(8.9)$ & 0.317 \\
\hline Agree/strongly agree & $57(86.4)$ & $112(91.1)$ & \\
\hline \multicolumn{4}{|c|}{ Smoking negatively impacts cancer treatment and survivorship } \\
\hline Completely disagree/disagree/no opinion & $6(9.1)$ & $11(8.9)$ & 0.960 \\
\hline Agree/strongly agree & $60(90.9)$ & $113(91.1)$ & \\
\hline \multicolumn{4}{|c|}{ Smoking cessation should be part of cancer treatment } \\
\hline Completely disagree/disagree/no opinion & $9(13.9)$ & $11(8.9)$ & 0.291 \\
\hline Agree/strongly agree & $56(86.1)$ & $113(91.1)$ & \\
\hline \multicolumn{4}{|l|}{ I have adequate smoking cessation training } \\
\hline Completely disagree/disagree/no opinion & $57(86.4)$ & $82(66.1)$ & 0.003 \\
\hline Agree/strongly agree & $9(13.6)$ & $42(33.9)$ & \\
\hline \multicolumn{4}{|c|}{ Healthcare providers should be aware of new and emerging tobacco products } \\
\hline Completely disagree/disagree/no opinion & $12(18.5)$ & $18(14.6)$ & 0.496 \\
\hline Agree/strongly agree & $53(81.5)$ & $105(85.4)$ & \\
\hline \multicolumn{4}{|l|}{ Healthcare providers should not smoke } \\
\hline Completely disagree/disagree/no opinion & $13(19.7)$ & 20 (16.3) & 0.553 \\
\hline Agree/strongly agree & $53(80.3)$ & $103(83.7)$ & \\
\hline
\end{tabular}

*Due to missing data, not all the variables add up to the total sample.

IDC, Instituto de Cancerología; INCan, Instituto Nacional de Cancerología.

\section{Knowledge about tobacco use}

The vast majority of CCPs at IDC (86.4\%) and INCan $(91.1 \%)$ agreed or strongly agreed that tobacco use is the major cause of lung cancer (table 2). Additionally, most of the respondents in Colombia $(90.9 \%)$ and Mexico $(91.1 \%)$ believe that it negatively impacts cancer treatment, as well as survivorship, and that smoking cessation services should be an integral part of cancer treatment (IDC $86.1 \%$; INCan $91.1 \%$ ). At the same time, around three quarters of the IDC (86.4\%) and at INCan CCPs (66.1\%) participating in our study considered to have inadequate training in smoking cessation, while $81.5 \%$ and $85.4 \%$ of the CCPs at IDC and INCan believed there should be more awareness of the new and emerging types of tobacco products. The majority of the CCPs at IDC $(80.3 \%)$ and INCan $(83.7 \%)$ believed that healthcare providers should not smoke.

\section{Smoking cessation practices among CCPs}

During patients' initial visit (table 3 ), approximately twothirds of the respondents at IDC $(67.5 \%)$ and INCan $(63.9 \%)$ reported always or most of the time asking patients about tobacco use. However, $48.6 \%$ and $46.7 \%$ of the participating CCPs at IDC and INCan, respectively, always or most of the time counsel their patients with cancer to quit. A lower proportion of CCPs always or most of the time provides any type of smoking cessation treatment or refer their patients to smoking cessationspecialised services (IDC 13.5\%, INCan 31.6\%).
During the follow-up visits of their patients with cancer, more than half of CCPs at IDC $(57.5 \%)$ and INCan $(59.3 \%)$ asked always or most of the time about tobacco use, $57.5 \%$ of CCPs at IDC and $52.2 \%$ of CCPs at INCan ask whether the patient had quit smoking, and $67.6 \%$ of CCPs at IDC and $59.7 \%$ of CCPs at INCan emphasise the importance of quitting to their patients with cancer during follow-up visits.

\section{Barriers to providing smoking cessation services}

The three most relevant barriers reported by the CCPs at IDC were (1) lack of local resources or referral centres for smoking cessation, (2) lack of training in smoking cessation and (3) patient resistance in quitting smoking (more details in table 4). The three most relevant barriers for providing smoking cessation services reported by the CCPs at INCan were (1) patient resistance in quitting smoking, (2) difficulties for motivating cancer patients to quit smoking and (3) lack of training in smoking cessation.

Determinants of providing cessation care or referral to patients with cancer at the initial visit

The logistic regression models assessing CCPs' factors associated with providing cessation treatment or referral to more specialised programmes are presented in table 5 . The resulting regression model for IDC did not retain statistically significant variables. CCPs appointed at INCan were less likely to provide cessation treatment or referral 
Table 3 Smoking cessation practices implemented by cancer care providers $(n=238)^{\star}$

\begin{tabular}{|c|c|c|c|}
\hline Practice & IDC n (\%) & INCan n (\%) & $P$ value \\
\hline \multicolumn{4}{|l|}{ During the initial visit } \\
\hline \multicolumn{4}{|c|}{ Ask cancer patients about tobacco use } \\
\hline Never/rarely/sometimes & $25(32.5)$ & $43(36.1)$ & 0.598 \\
\hline Most times/always & $52(67.5)$ & $76(63.9)$ & \\
\hline \multicolumn{4}{|c|}{ Counsel cancer patients to quit } \\
\hline Never/rarely/sometimes & $37(51.4)$ & $64(53.3)$ & 0.794 \\
\hline Most times/always & $35(48.6)$ & $56(46.7)$ & \\
\hline \multicolumn{4}{|c|}{ Provide treatment/refer cancer patients to quit } \\
\hline Never/rarely/sometimes & $64(86.5)$ & $80(68.4)$ & 0.005 \\
\hline Most times/always & $10(13.5)$ & $37(31.6)$ & \\
\hline \multicolumn{4}{|l|}{ During follow-up visits } \\
\hline \multicolumn{4}{|c|}{ Ask cancer patients about tobacco use } \\
\hline Never/rarely/sometimes & $31(42.5)$ & $46(40.7)$ & 0.812 \\
\hline Most times/always & $42(57.5)$ & $67(59.3)$ & \\
\hline \multicolumn{4}{|l|}{ Ask if patient had quit } \\
\hline Never/rarely/sometimes & $31(42.5)$ & $54(47.8)$ & 0.477 \\
\hline Most times/always & $42(57.5)$ & $59(52.2)$ & \\
\hline \multicolumn{4}{|c|}{ Emphasise to patients the importance of quitting } \\
\hline Never/rarely/sometimes & $24(32.4)$ & $46(40.4)$ & 0.273 \\
\hline Most times/always & $50(67.6)$ & $68(59.7)$ & \\
\hline
\end{tabular}

${ }^{*}$ Due to missing data, not all the variables add up to the total sample.

IDC, Instituto de Cancerología; INCan, Instituto Nacional de Cancerología.

to their patients if they had less than $50 \%$ of their time devoted to patient care (OR $0.24 ; 95 \%$ CI 0.06 to 0.88 ; $\mathrm{p}=0.03$ ) and were former or current smokers (OR 0.31; $95 \%$ CI 0.1 to $0.88 ; \mathrm{p}=0.03$ ).

\section{DISCUSSION}

While the prevalence of infectious diseases in Latin America is reducing rapidly, lifestyle changes such as increased cigarette smoking in the region are associated with the rising trend of non-communicable diseases, including cancer. ${ }^{18}{ }^{19}$ Epidemiological reports have highlighted the public health burden and economic cost of cancer in Latin America. ${ }^{20} 21$ In the region, about 1.3 million new cancer cases and 666000 cancer deaths occurred in 2018. The leading cancers diagnosed are breast, prostate, colorectal, lung and stomach cancer, with lung cancer as the leading cause of death. ${ }^{22}$ Cigarette smoking is a modifiable cancer risk factor that adversely impacts treatment outcomes and quality of life if it continues following a cancer diagnosis. ${ }^{23}$ Despite the publication of national smoking cessation guidelines for health professionals in Colombia ${ }^{24}$ and Mexico, ${ }^{25-27}$ patients in these countries who continue smoking after a cancer diagnosis have limited access to evidence-based interventions or resources that assist them in their quitting efforts.
In a 2019 systematic review and meta-analysis conducted among 57415 physicians, dentists, nurses and pharmacists in 63 countries, the overall prevalence of tobacco use (cigarettes, water pipe, smokeless tobacco) was $21 \% .^{8}$ In the same review, the pooled prevalence among healthcare professionals in lower middle-income and low-income countries was 19\%. In our study, current cigarette smoking prevalence among participating CCPs in Colombia and Mexico was $10.5 \%$ and $12.3 \%$, respectively. While these rates may seem low, it is important to consider that smoking prevalence for the general population by the time when the study was conducted was $11.1 \%$ in Colombia and $11.9 \%$ in Mexico. ${ }^{28}$ Social desirability bias may be associated with underreporting of current cigarette smoking in our study sample, leading some CCPs to report that they were former smokers. When combining prevalence rates of current and former smoking, these notable fractions represent $32.9 \%$ and $46.7 \%$ of the participating CCPs in Colombia and Mexico, respectively. Additionally, $19.7 \%$ of the surveyed CCPs in Colombia and $16.3 \%$ of those in Mexico do not believe that healthcare providers should not smoke. When assessing determinants for providing cessation care or referral services to patients with cancer at their initial visit, we found that being a current or former smoker and having less than $50 \%$ of dedicated time to patient care could undermine 
Table 4 Respondents' barriers to providing smoking cessation services $(n=238)^{*}$

\begin{tabular}{|c|c|c|c|}
\hline Barriers & $\begin{array}{l}\text { IDC } \\
\text { n (\%) }\end{array}$ & $\begin{array}{l}\text { INCan } \\
\text { n (\%) }\end{array}$ & $P$ value \\
\hline \multicolumn{4}{|c|}{ Difficulties for motivating cancer patients to quit smoking } \\
\hline Completely disagree/disagree/no opinion & $56(66.7)$ & $42(35.9)$ & 0.001 \\
\hline Agree/strongly agree & $28(33.3)$ & $75(64.1)$ & \\
\hline \multicolumn{4}{|c|}{ Implementing smoking cessation among cancer patients is a waste of time } \\
\hline Completely disagree/disagree/no opinion & 77 (90.6) & $88(75.2)$ & 0.005 \\
\hline Agree/strongly agree & $8(9.4)$ & $29(24.8)$ & \\
\hline \multicolumn{4}{|c|}{ Lack of time for implementing smoking cessation counselling } \\
\hline Completely disagree/disagree/no opinion & $34(40.0)$ & $51(43.97)$ & 0.574 \\
\hline Agree/strongly agree & $51(60.0)$ & $65(56.03)$ & \\
\hline \multicolumn{4}{|c|}{ Lack of reimbursement for implementing smoking cessation counselling } \\
\hline Completely disagree/disagree/no opinion & $65(77.38)$ & $88(75.86)$ & 0.803 \\
\hline Agree/strongly agree & $19(22.62)$ & $28(24.14)$ & \\
\hline \multicolumn{4}{|l|}{ Cancer patient resistance in quitting smoking } \\
\hline Completely disagree/disagree/no opinion & $26(31.33)$ & $31(26.5)$ & 0.456 \\
\hline Agree/strongly agree & $57(68.67)$ & $86(73.5)$ & \\
\hline \multicolumn{4}{|l|}{ Lack of training in smoking cessation } \\
\hline Completely disagree/disagree/no opinion & $20(23.53)$ & $42(36.21)$ & 0.055 \\
\hline Agree/strongly agree & $65(76.47)$ & $74(63.79)$ & \\
\hline \multicolumn{4}{|c|}{ Lack of local resources or referral centres for smoking cessation } \\
\hline Completely disagree/disagree/no opinion & $17(20.24)$ & $50(43.1)$ & 0.001 \\
\hline Agree/strongly agree & $67(79.76)$ & $66(56.9)$ & \\
\hline \multicolumn{4}{|l|}{ Costs of smoking cessation treatment } \\
\hline Completely disagree/disagree/no opinion & $37(43.53)$ & $61(52.14)$ & 0.227 \\
\hline Agree/strongly agree & $48(56.47)$ & $56(47.86)$ & \\
\hline \multicolumn{4}{|l|}{ Lack of smoking cessation medication } \\
\hline Completely disagree/disagree/no opinion & $34(40.0)$ & $47(40.17)$ & 0.980 \\
\hline Agree/strongly agree & $51(60.0)$ & $70(59.83)$ & \\
\hline \multicolumn{4}{|l|}{ Tobacco use by healthcare professionals } \\
\hline Completely disagree/disagree/no opinion & $53(62.35)$ & $52(44.44)$ & 0.012 \\
\hline Agree/strongly agree & $32(37.65)$ & $65(55.56)$ & \\
\hline \multicolumn{4}{|c|}{ Marketing of new and emerging tobacco products as a healthier alternative for smoking cessation } \\
\hline Completely disagree/disagree/no opinion & $47(56.63)$ & 46 (39.32) & 0.016 \\
\hline Agree/strongly agree & $36(43.37)$ & $71(60.68)$ & \\
\hline
\end{tabular}

*Due to missing data, not all the variables add up to the total sample.

IDC, Instituto de Cancerología; INCan, Instituto Nacional de Cancerología.

smoking cessation efforts in oncology settings. However, these results should be interpreted with caution because these statistically significant associations only were found among CCPs at INCan.

These findings only highlight how smoking behaviour among CCPs and the normalisation of the tobacco culture at IDC and INCan are major challenges to overcome for implementing effective smoking cessation services in these oncology centres. ${ }^{92}$ Our study also found that $13.6 \%$ and $33.9 \%$ of the CCPs at IDC and INCan felt that they were not adequately trained to treat tobacco dependence or provide referral options to their patients, while the vast majority of our study participants $(90.9 \%$ and $91.1 \%$ of the CCPs at IDC and INCan, respectively) believed that smoking cessation should be a standard component of oncologic treatment. In agreement with previous reports, ${ }^{9}$ our study data also suggest that CCPs are more likely to 'ask' and 'advise' patients with cancer about their tobacco use during the initial visit and less likely to proactively 'assist' them with their quitting efforts. 
Table 5 Multivariate analysis for the relationship of CCPs' characteristics with the provision of smoking cessation treatment or referral at initial visit

\section{Significant relationship}

Adjusted OR

$95 \% \mathrm{Cl}$

$P$ value

IDC

Model 1: provide cessation treatment or referral

$\mathrm{N} / \mathrm{A}^{*}$

INCan

Model 2: provide cessation treatment or referral

Time percentage with patient care, $\leq 50 \%$ vs $>50 \%$ (ref)

Being former or current smoker, yes vs no (ref)

0.2

$0.1-0.9$

0.031

0.3

$0.1-0.9$

0.028

${ }^{*}$ No variable with statistically significant association retained in the model.

†Variables included in the models: age, years since graduation, gender, years since graduation, professional degree, time percentage with devoted to patient care, smoking status, knowledge of any local resources or services that assist patients with quitting smoking, lack of training in smoking cessation, lack of local resources or referral and patient resistance in quitting smoking.

CCP, cancer care provider; IDC, Instituto de Cancerología; INCan, Instituto Nacional de Cancerología.

During the follow-up appointments, more than a third of the surveyed CPPs at IDC and INCan failed to consistently address tobacco use by their patients. These findings clearly showed that many opportunities for smoking cessation support were missed at IDC and INCan.

Our results are mostly limited to the cross-sectional design of our study and self-reported data, which are subjected to bias. Also, our findings are not generalisable as our sample was restricted to only two cancer centres in Latin America. Data from the participating cancer centres are not comparable as IDC is a private institution, and INCan is a national cancer centre, each with different policies, oversight bodies and resources. Nevertheless, this is among the first reports assessing characteristics, tobacco use (including e-cigarettes) and smoking cessation practices among Latin American CCPs.

Additionally, the response rate among CCPs at INCan was low $(17.5 \%)$. It may be possible that only those CCPs at INCan who were interested and/or sensitised towards smoking cessation responded to our survey. We believe that originating the invitational emails and reminders from the IDC director's office yielded a very satisfactory response rate at this institution $(83.9 \%)$. While only individuals providing direct care to patients with cancer were included in the study, there was an important proportion of CCPs who had less than $50 \%$ time devoted to cancer care $(29.4 \%)$. Limited opportunities for interacting with patients could have impacted study findings related to CCPs' practices for promoting smoking cessation. This study set a foundation for subsequent qualitative methods that can shed light on facilitators of and barriers to integrate smoking cessation into oncology services. Also, quantitative research with broader samples will help to determine the statistical associations between qualitatively identified factors and outcomes for implementing smoking cessation approaches.

While few and/or inconsistent educational programmes for smoking cessation have been developed in Spanish for healthcare professionals in Latin America, ${ }^{30-34}$ training opportunities specifically tailored to CCPs are lacking in the region for effectively influencing smoking behaviours in patients with cancer and survivors. High levels of mental health disorders, numerous quit attempts, complex medication regimens, potential side effects, drug interactions, economic burden and scheduling conflict are among the multiple challenges to be considered when delivering smoking cessation interventions to patients with cancer. ${ }^{35}$ Based on our findings, such training programmes tailored to CCPs should build relevant skills and knowledge for: (1) including standard tobacco use definitions in the medical record of patients with cancer, (2) addressing cancer patient resistance in quitting smoking, (3) motivating patients with cancer to quit smoking, (4) creating local resources or identifying referral centres for smoking cessation and 5) considering the tailored pharmacotherapy options for smoking cessation during cancer treatment.

While our findings can contribute to the successful design and implementation of smoking cessation programmes in oncology settings, it is also important to consider a wide range of additional factors. The socioecological model could be used as a framework for this purpose as it would address not only individual CCPs' characteristics and attitudes but also patient-provider relationships, in addition to organisational, community and societal factors that shape the adoption and implementation of sustainable smoking cessation interventions. ${ }^{36}$

Finally, $8.7 \%$ of the CCPs at IDC and $7.6 \%$ of the CCPs at INCan participating in our study were either current or former users of e-cigarette. Considering the overall market growth of these new products in Latin America, the potential of e-cigarette use as a harm reduction strategy or cessation tool is worth mentioning as it remains controversial within the scientific community. A randomised trial conducted in the UK among 886 adult smokers suggests that e-cigarettes may be more effective than nicotine-replacement therapy for smoking cessation. ${ }^{37}$ However, 'Switching to e-cigarettes does not mean 
quitting. ... Quitting means truly ending the addiction to nicotine' according to The American Lung Association. ${ }^{38}$ Therefore, Latin American healthcare professionals, researchers and policymakers should proceed with caution when considering e-cigarettes as a harm reduction alternative to smoking until the long-term health effects of these new emerging products are well established.

Integrating smoking cessation services in Latin American oncology settings not only would improve treatment outcomes and survivorship for patients with cancer in the region but will be determinant for expanding the comprehensive role of these cancer centres from cancer diagnosis and treatment towards including cancer prevention and control initiatives along the cancer care continuum.

\section{Author affiliations}

${ }^{1}$ Department of Epidemiology, Human Genetics, and Environmental Sciences, University of Texas Health Science Center at Houston, Houston, Texas, USA ${ }^{2}$ Institutional Liaison Department, Instituto Nacional de Cancerologia, Mexico, DF, Mexico

${ }^{3}$ Department of Tobacco Research, Instituto Nacional de Salud Publica, Cuernavaca, Morelos, Mexico

${ }^{4}$ Tobacco Smoking and COPD Research Department, Instituto Nacional de Enfermedades Respiratorias, Mexico, DF, Mexico

${ }^{5}$ Division of Cancer Prevention and Population Sciences, University of Texas MD Anderson Cancer Center, Houston, Texas, USA

${ }^{6}$ Research Unit, Instituto de Cancerologia, Medellin, Antioquia, Colombia

Acknowledgements Authors thanks MD Anderson Assessment, Intervention and Measurement (AIM) core for assisting with data management. Authors would also like to express their gratitude to Luis Herrera, $\mathrm{PhD}$ at the Instituto Nacional de Cancerología, México for facilitating the local logistics and fruitful partnership with researchers at the Instituto Nacional de Enfermedades Respiratorias, Mexico, and the Instituto Nacional de Salud Pública, Mexico, which secured the successful execution of our project in this country. No patient was involved in our research study.

Contributors IT-M conceived the study, was in charge of overall direction and planning, and took the lead in writing the manuscript; LMR-S and LG contributed to the study design and development of data collection instrument; LS, carried out recruitment and data collection procedures in Mexico; $A B$ and $\mathrm{HG}$ carried out recruitment and data collection procedures in Colombia; MC and SS analysed the data; PMC and EH provided critical feedback and helped shape the research. All authors contributed to the interpretation of the results and contributed to the final manuscript.

Funding This research was supported in part by the National Institutes of Health through a Cancer Center Support Grant (P30CA16672) to The University of Texas MD Anderson Cancer Center, USA; the MD Anderson Sister Institute Network Fund Award to principal investigator I. Tamí-Maury, DMD, MSc, Dr.PH; The Hope Foundation Impact Award to principal investigator I. Tamí-Maury, DMD, MSc, Dr.PH; the Secretary of Health of Medellin City, Colombia (460005975); and the National Cancer Institute, Mexico.

Competing interests None declared.

Patient and public involvement Patients and/or the public were not involved in the design, or conduct, or reporting, or dissemination plans of this research.

Patient consent for publication Not required.

Ethics approval This study was approved by the Ethics Boards of IDC (Protocol Number: 02--2015-I), INCan (Protocol Number: 016/001/DII; CEI/994/16), and MD Anderson Cancer Center (Protocol Number: PA14-1060).

Data availability statement № data are available. No data are available.

Open access This is an open access article distributed in accordance with the Creative Commons Attribution Non Commercial (CC BY-NC 4.0) license, which permits others to distribute, remix, adapt, build upon this work non-commercially, and license their derivative works on different terms, provided the original work is properly cited, appropriate credit is given, any changes made indicated, and the use is non-commercial. See: http://creativecommons.org/licenses/by-nc/4.0/.

ORCID iD

Irene Tami-Maury http://orcid.org/0000-0002-1404-8641

\section{REFERENCES}

1 Goss PE, Lee BL, Badovinac-Crnjevic T, et al. Planning cancer control in Latin America and the Caribbean. Lancet Oncol 2013:14:391-436.

2 Thun M, Peto R, Boreham J, et al. Stages of the cigarette epidemic on entering its second century. Tob Control 2012;21:96-101.

3 Who report on the global tobacco epidemic, 2019

4 Salloum RG, Huo J, Lee J-H, et al. Tobacco and e-cigarette use among cancer survivors in the United States. PLoS One 2019;14:e0226110-e.

5 Parsons A, Daley A, Begh R, et al. Influence of smoking cessation after diagnosis of early stage lung cancer on prognosis: systematic review of observational studies with meta-analysis. BMJ 2010;340:b5569.

6 Graham W, Toll B, Tamí-Maury I, et al. Cancer prevention: Tobacco dependence and its treatment. In: DeVita J VT, Lawrence TS, Rosenberg SA, eds. DeVita, Hellman, and Rosenberg's Cancer: Principles \& Practice of Oncology. Philadelphia: Lippincott Williams \& Wilkins, 2014.

7 Gritz ER, Toll BA, Warren GW. Tobacco use in the oncology setting: advancing clinical practice and research. Cancer Epidemiol Biomarkers Prev 2014;23:3-9.

8 Nilan K, McKeever TM, McNeill A, et al. Prevalence of tobacco use in healthcare workers: a systematic review and meta-analysis. PLoS One 2019;14:e0220168-e.

9 Warren GW, Marshall JR, Cummings KM, et al. Practice patterns and perceptions of thoracic oncology providers on tobacco use and cessation in cancer patients. J Thorac Oncol 2013;8:543-8.

10 Stead LF, Buitrago D, Preciado N, et al. Physician advice for smoking cessation. Cochrane Database Syst Rev 2013;5:Cd000165.

11 Müller F, Wehbe L. Smoking and smoking cessation in Latin America: a review of the current situation and available treatments. Int $J$ Chron Obstruct Pulmon Dis 2008;3:285-93.

12 Abdullah A, Stillman F, Yang L, et al. Tobacco use and smoking cessation practices among physicians in developing countries: a literature review (1987-2010). Int J Environ Res Public Health 2014;11:429-55

13 Sansores $\mathrm{RH}$, Villalba-Caloca J, Herrera-Kiengelher L, et al. Prevalence of cigarette smoking among employees of the Mexican National Institutes of health. Salud Publica Mex 1999;41:381-8.

14 González-Zapata LI, Deossa GC, Monsalve-Álvarez J, et al. Metabolic syndrome in healthcare personnel of the University of Antioquia-Colombia; LATINMETS study. Nutr Hosp 2013;28:522-31.

15 Payne TJ, Gaughf NW, Sutton MJ, et al. The impact of brief tobacco treatment training on practice behaviours, self-efficacy and attitudes among healthcare providers. Int J Clin Pract 2014;68:882-9.

16 The GATS atlas: global adult tobacco survey: World Health organization, 2015. Available: https://www.who.int/tobacco/ publications/surveillance/gatstlas/en/

17 Tibshirani R. Regression shrinkage and selection via the LASSO. Journal of the Royal Statistical Society 1996;58:267-88.

18 Boutayeb A, Boutayeb S. The burden of non communicable diseases in developing countries. Int J Equity Health 2005;4:2.

19 De Maio FG. Understanding chronic non-communicable diseases in Latin America: towards an equity-based research agenda. Global Health 2011;7:36.

20 Sierra MS, Forman D. Cancer in central and South America: methodology. Cancer Epidemiol 2016;44:S11-22.

21 Bray F, Piñeros M. Cancer patterns, trends and projections in Latin America and the Caribbean: a global context. Salud Publica Mex 2016:58:104-17.

22 Prostate. Breast and colorectal cancer are the main cancers in the region: the cancer atlas, 2018. Available: https://canceratlas.cancer. org/the-burden/latin-america-and-the-caribbean/

23 Jassem J. Tobacco smoking after diagnosis of cancer: clinical aspects. Trans/ Lung Cancer Res 2019;8:S50-8.

24 Cesación Tabáquica. Compilación de las recomendaciones actualizadas para profesionales de la salud en Colombia. Bogota: Instituto Nacional de Cancerología - ESE, 2016.

25 Tabaco: Prevención de adicciones, 2000

26 Guías Mexicanas para El Tratamiento del Tabaquismo. Un Consenso Nacional de Expertos Neumol Cir Torax 2005;64:84-112. 
27 Prevención. diagnóstico $Y$ tratamiento del consumo de tabaco $Y$ humo ajeno, en El primer nivel de atención. Mexico City: Centro Nacional de Excelencia Tecnológica en Salud, 2009.

28 Drope J, Schluger N, Cahn Z, et al. The tobacco atlas Atlanta: American cancer Society and vital strategies, 2018. Available: https:// tobaccoatlas.org/

29 Warren GW, Marshall JR, Cummings KM, et al. Addressing tobacco use in patients with cancer: a survey of American Society of clinical oncology members. J Oncol Pract 2013;9:258-62.

30 Tabáquica C. Curso virtual Cali: Universidad ICESI, 2020. Available: https://www.icesi.edu.co/educacion_continua/cursos-virtuales/ curso-virtual-en-cesacion-tabaquica

31 Curso Virtual sobre "Abordaje Integral del Tratamiento del Tabaquismo" Buenos Aires: Asociación Toxicológica Argentina, 2018. Available: https://toxicologia.org.ar/curso-virtual-abordaje-integraldel-tratamiento-del-tabaquismo-segunda-edicion-ano-2018/

32 Virtual course on tobacco control Washington, D.C.: pan American health organization, 2010. Available: https://www.paho.org/hq/index. php?option=com_content\&view=article\&id=3417:virtual-course-ontobacco-control\&ltemid=1185\&lang=en $3311^{\circ}$ Curso de capacitación $\mathrm{Y}$ formación en tabaquismo para profesionales de la salud Buenos Aires: Asociación Argentina de medicina Respiratoria, 2020. Available: http://www.uata.org.ar/ 10o-curso-de-capacitacion-y-formacion-en-tabaquismo-paraprofesionales-de-la-salud.html

34 Martínez C, Castellano Y, Company A, et al. Impact of an online training program in hospital workers' smoking cessation interventions in Bolivia, Guatemala and Paraguay. Gac Sanit 2018;32:236-43.

35 Karam-Hage M, Oughli HA, Rabius V, et al. Tobacco cessation treatment pathways for patients with cancer: 10 years in the making. J Natl Compr Canc Netw 2016;14:1469-77.

36 Allen J. A Socioecological approach to addressing tobacco related health disparities. Bethesda, MD: National Cancer Institute, 2017Report No.: NIH Publication No. 17-CA-8035A

37 Hajek P, Phillips-Waller A, Przulj D, et al. A randomized trial of e-cigarettes versus Nicotine-Replacement therapy. N Engl J Med Overseas Ed 2019;380:629-37.

38 LaMotte S. Uk study shows e-cigarettes help adult smokers quit, but us experts urge caution, 2019 